\title{
Identification of Potential Antibody Epitopes in MMP-15
}

\author{
Jessie Gan ${ }^{1}$, Cheryl Eisen ${ }^{1}$, Vaughn Smider ${ }^{2,3} *$ \\ ${ }^{1}$ San Diego Jewish Academy, San Diego, CA 92130 \\ ${ }^{2}$ Applied Biomedical Science Institute, San Diego, CA 92127 \\ *Corresponding author: Vaughn Smider \\ E-mail: vsmider@absinstitute.org \\ Running title: MMP-15 Epitopes
}

${ }^{3}$ The Scripps Research Institute, Dept. of Molecular Medicine, La Jolla, CA, 92037

Keywords: Immunotherapy, bioinformatics, matrix metalloproteinases, epitopes, monoclonal antibody, drug development 


\begin{abstract}
Monoclonal antibody therapy is a well-established cancer treatment paradigm that often targets cancerspecific cell surface proteins. Matrix Metalloproteinase 15 (MMP-15) is a surface protein implicated in metastasis and angiogenesis, however it is not well characterized. Here we use bioinformatics tools to identify epitopes for drug or diagnostic targeting and elucidate features of this potentially important protein in metastatic processes. We identified conserved regions in MMP-15 as well as unique, variable peptide regions compared to other Membrane Type MMPs (MT-MMP). Conservation of catalytic and hemopexin domains in MMP-15 imply functional importance, however, their similarity to other MT-MMPs discourage use as target epitopes. Our analyses also identified an MMP-15 peptide that was highly divergent from other MMPs, suggesting that it may serve as an appropriate specific epitope for a specific antibody drug. Thus, we were able to elucidate features and potential unique epitopes of MMP-15 for use in further antibody discovery and targeting.
\end{abstract}

\title{
Introduction
}

Lung cancer is the number one cause of cancer-related deaths in industrialized countries. The expected 5year survival rate in lung cancer is $15 \%$, compared to the over $80 \% 5$-year survival rates of breast and prostate cancers. Furthermore, $85 \%$ of diagnosed lung cancers are non-small-cell-lung cancer (NSCLC) (Tyagi) where recent advances in novel targeted therapies have not yet made a major impact on patient survival. Combinations of conventional chemotherapy, radiation, and surgery are the standard treatment for NSCLC; however, they haven't significantly improved prognosis since 1975 (Tyagi). Immunotherapy is a novel modality that utilizes the power of the human immune system to eliminate cancer cells. Types of immunotherapies include monoclonal antibodies, antibody drug conjugates, cancer vaccines, and chimeric antigen receptor - $\mathrm{T}$ cells (CAR-T). These therapies function by blocking proliferation signals, recruiting lymphocytes to the tumor, developing an immune memory of tumor antigens, and targeting cytotoxins for immediate cytotoxicity (Hallam, Dotti, Ho). The linchpin of immunotherapy is the unique antigenspecificity that can distinguish between normal and cancerous cells, allowing treatment to be both potent and specific. Antigen and epitope identification is crucial to the drug development process; finding cancerspecific antigen epitopes of interest an essential challenge in the development of this therapy (Steven).

Matrix Metalloproteinases (MMPs) are zinc-dependent enzymes that proteolyze extracellular matrix (ECM). There are 24 MMPs identified in humans, rodents, and amphibians that share structural elements such as a pro-peptide of about 80 amino acids, a catalytic domain of about 170 amino acids, a linker peptide of various lengths, and a hemopexin domain of about 200 amino acids, with a few exceptions (Cathcart, Nagase). In humans, MMPs are concentrated in connective tissue and epithelial cells. Their normal function is breaking down various extracellular matrix molecules or cleaving specific proteins (Nagase, Araki).

MMP-15 is a type-1 transmembrane protein. The transmembrane MMPs (MT-MMPs) share a common structure of a signal peptide, a peptidoglycan domain, a cysteine switch, a catalytic domain, a hinge (linker 1), a hemopexin-like domain (Hpx), a stalk region (linker 2), transmembrane region, and cytoplasmic region. In addition, transmembrane MT-MMPs also have a unique insertion of 8-9 amino acids in their catalytic domain known as the MT-loop (Itoh, Araki). 
Activation of TM-MMPs involve proproteins, also known as protein convertases (PCs). The sequence $\mathrm{RXKR}$ in the proprotein domain is the $\mathrm{PC}$ recognition sequence, and $\mathrm{PCs}$ such as furin cleave this site while the protein is still in the ER/Golgi (Itoh). The PC cleavage is dependent on the zinc binding motif in the catalytic domain and cysteine switch motif in the propeptide, which prevent proMMPs from being activated intracellularly. The zinc binding motif of HEXXHXXGXXH and the cysteine switch motif of PRCGXPD are in a Cys- $\mathrm{Zn}^{2+}$ coordination, preventing an essential water molecule for catalysis from binding to the zinc ion, and blocking early activation within the cell (Itoh). As the propeptide is cleaved off, this connection is severed and the zinc ion is open for catalysis as the enzyme is presented on the cell surface (Nagase, Fanjul-Fernandez).

The catalytic domain is composed of a 5 -stranded $\beta$-sheet, three $\alpha$-helices, and a connective loop, along with other ions for stability. There are two zinc ions (one catalytic and one structural) and up to three structural calcium ions. Among MMPs, the catalytic domains are mostly conserved with the exception of a hydrophobic S1' pocket near the active site, which is the primary determinant of substrate specificity (Nagase, Loffek). To support the active site, the catalytic domains also contain a conserved methionine, which forms a "Met-turn" eight residues after the active domain. Aside from all MMPs, a unique feature of MT-MMP catalytic domains is an 8 amino acid loop insertion in the catalytic domain. This loop is integral for MT-MMPs' function in the activation of proMMP-2 (Nagase, Itoh).

MMPs can regulate tumor progression processes such as cell death, proliferation, differentiation, tumorassociated angiogenesis, and malignant conversion (Loffek, Nagase, Hotary2002). As a tumor grows, it requires reorganization of its environment. Cancer fuels its need for growth through breakdown of ECM by overexpressing proteolytic enzymes like MMP-15 (Dornier). MMP-15 can degrade collagen-I, but it is not considered a major collagenolytic enzyme. However, it can also degrade fibrin and promote cellular invasion into fibrin matrices and basement membranes, the extracellular matrix that underlies all epithelia and endothelia (Hotary2002, Hotary2000, Hotary2006, Ota, Löffek ). In addition, MMP-15 has been shown to proteolyze fibronectin, laminin-1, nidogen, perlecan, and $\mathrm{NC1}$ as substrates, all of which are ECM components. (Itoh, D’Ortho) However, MMP-15 also contributes to cancer promotion in other ways, such as through blocking Fas and TRAIL death ligands and associating with other MMPs. (Ito, Kobayashi, Abraham, Joshi, Liu)

We are interested in therapeutic monoclonal antibodies specific to upregulated cancer antigens for tumor targeting (Pento, Aldaroish). One such possible antigen is MMP-15, which is upregulated in NSCLC and may be involved in metastasis through ECM cleavage (Magdalena, Tao). We use the cumulative information from online databanks combined with bioinformatics, the collaborative synthesis of multiple analysis tools, to further investigate the MMP-15 protein for possible specific epitopes for antibody targeting.

\section{Results}

To assess the potentially viable epitopes of the MMP-15 protein for antibody targeting, we curated the MMP-15 sequences of several species as well as MT-MMP sequences. We employed multiple sequence alignment and analyzed various other features from the comparison such as percent identity and Shannon 
entropy. In addition, we utilized partial-MMP-14 crystal structures to analyze the catalytic (PDBID: 3MA2) and hemopexin (PDBID: 3C7X) domains. The overall workflow is depicted in Figure 1.

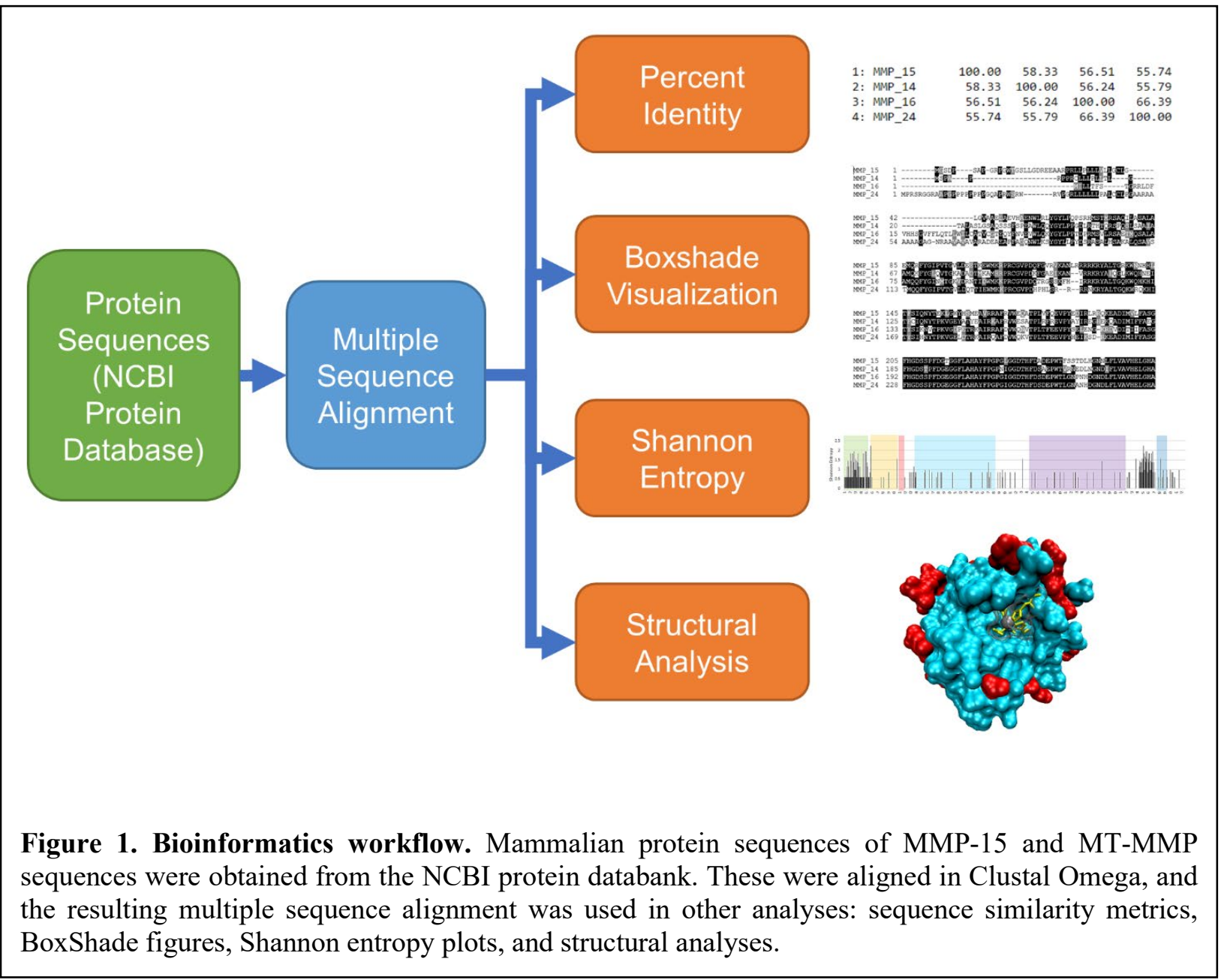

\section{Mammalian MMP-15 Ortholog Comparison}

We identified regions of similarity among MMP-15 sequences from various species in order to investigate regions of evolutionary conservation, which may imply their importance to functional mechanisms. Additionally, conserved epitope targeting would enable cross-reactivity of an experimental therapeutic in future animal efficacy or toxicity studies. From the multiple sequence alignment of mammalian orthologs, we found that on average $92.9 \%$ of MMP-15 amino acid residues are conserved across the aligned sequences. The enzyme active site is particularly highly conserved at an average identity of $94.7 \%$. The sequence similarity pairwise heatmaps can be found in Supplemental Figures 1 and 2, for the entire sequence and catalytic domain respectively, and the corresponding matrixes produced from Clustal Omega are in Supplemental Tables 1 and 2.

In the mammalian MMP-15 ortholog comparison, the most sequence variability, as evaluated by Shannon entropy analysis, is seen in the signal peptide as well as a region just $\mathrm{N}$-terminal to the transmembrane region (Figure 2A). 


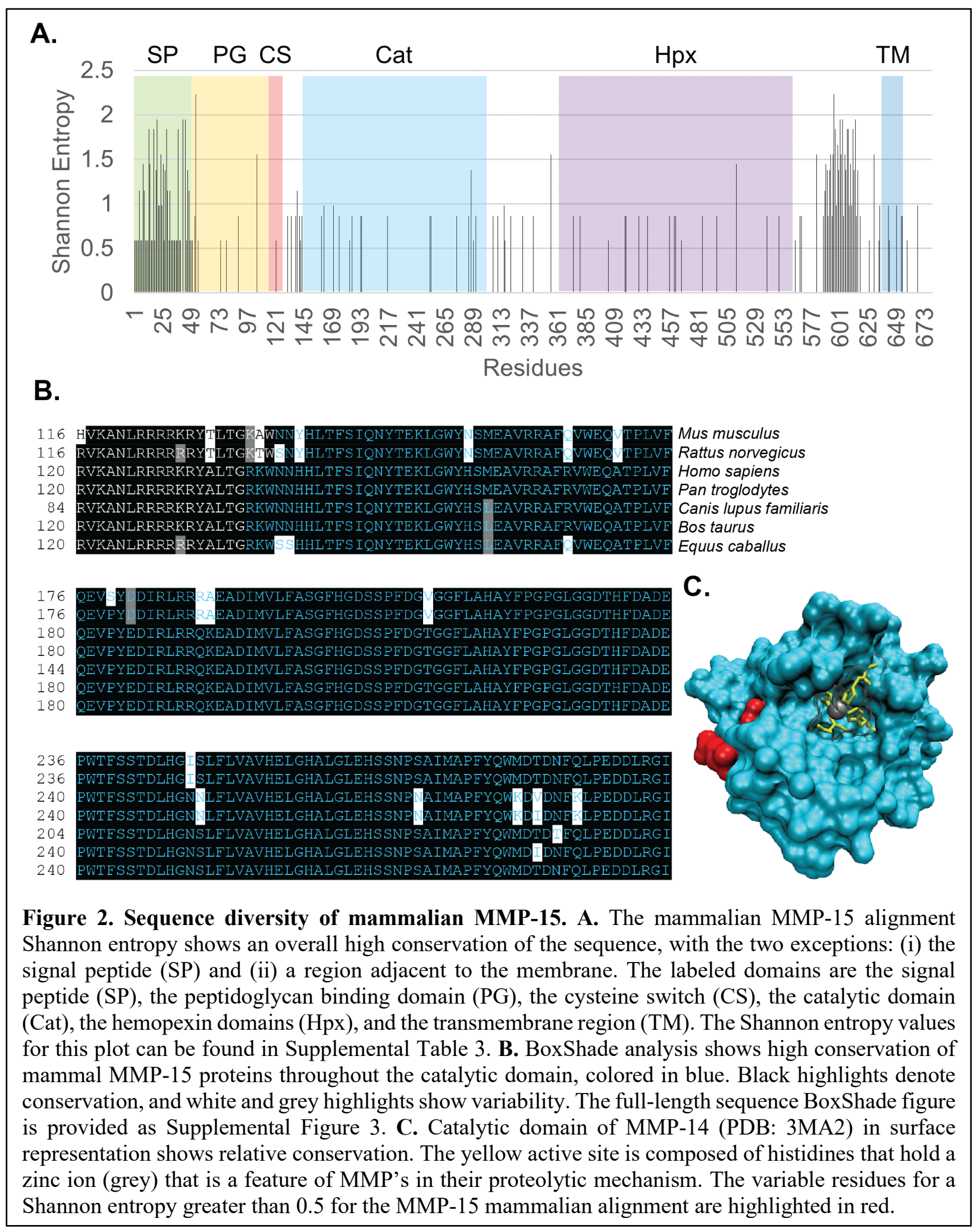


As shown in Figure 2B and 2C, the active site of the catalytic domain is highly conserved. Although there are a few variable residues in the catalytic domain, they are on the opposite side of the conserved active site. The two visible variable residues in Figure 2C are N114 and T78 (SE>0.5); all variable residues in the catalytic domain had low Shannon entropy scores near 1, indicating considerable conservation. In addition, structural analysis of the hemopexins additionally showed that this is a highly conserved domain (Supplemental Figure 4).

\section{Membrane-Type MMP Paralog Comparison}

In order to identify regions within MMP-15 that are unique compared to other MT-MMP's, we compared four human MT-MMP (MMP-14, 15, 16, and 24) paralog sequences in a multiple sequence alignment. We found that on average $56.9 \%$ of residues are conserved among the MMPs compared. The enzyme active site is more conserved at an average of $68.5 \%$. The sequence similarity heatmap across the entire sequence is provided in Supplemental Figure 5, with the corresponding matrix from Clustal Omega in Supplemental Table 4.
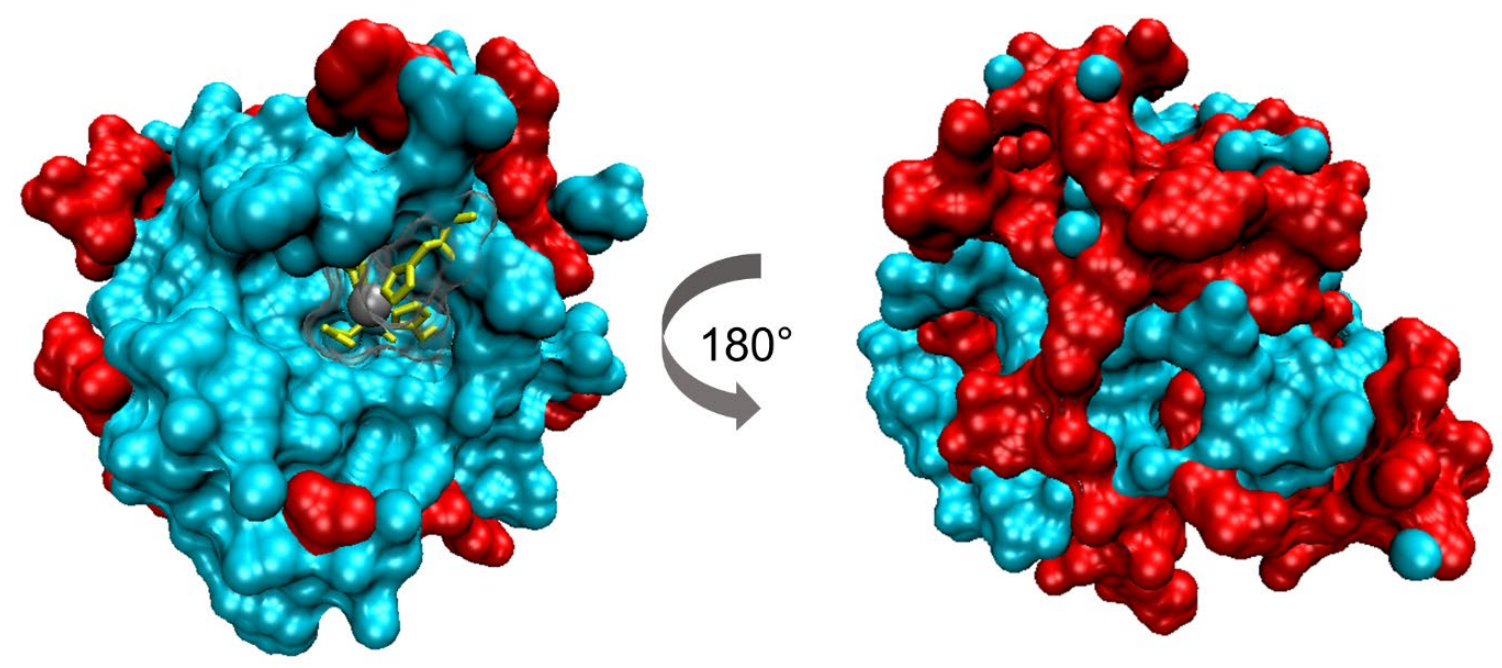

Figure 3. Structural diversity of MMP-15 catalytic domain compared to other MT-MMPs. The MT-MMP alignment reveals conserved and variable regions of the catalytic. The catalytic domain of crystal MMP-14 is in surface representation. The yellow active site is composed of histidines that hold a zinc ion (grey) that is a feature of MMP's in their proteolytic mechanism. The variable residues of the MT-MMP alignment based on Shannon entropy values ranging from 0.8 to 1.5 are highlighted in red.

As there is currently no structural information on MMP-15 itself, we used the crystal structure of the related MMP-14 catalytic domain as a surrogate to visualize structural diversity. The catalytic domain has clear areas of structural conservation, as well as more variable regions than the mammalian alignment (Figure 3 ). However, the majority of the variable residues are on the side opposite the active site, leaving the active site itself, and residues surrounding it, mostly conserved. Thus, the face of the enzyme containing the active stie is highly conserved whereas the opposite face appears much more mutable. In addition, compared to 
the hemopexin domains in the mammalian MMP-15 alignment, the hemopexin domains of the multiple MT-MMP alignment are also much more variable (Supplemental Figure 7).

According to the Shannon entropy results (Figure 4), the MT-MMP paralog alignment shows more variability than the mammalian alignment of MMP-15 orthologs. In two select areas within the catalytic domain of paralogs, there is nearly a complete conservation of residues, whereas in other domains there is no such conservation. In addition to revealing the most conserved regions, Shannon entropy analysis also identifies the most variable regions, which may be of interest as novel antibody targeting epitopes.

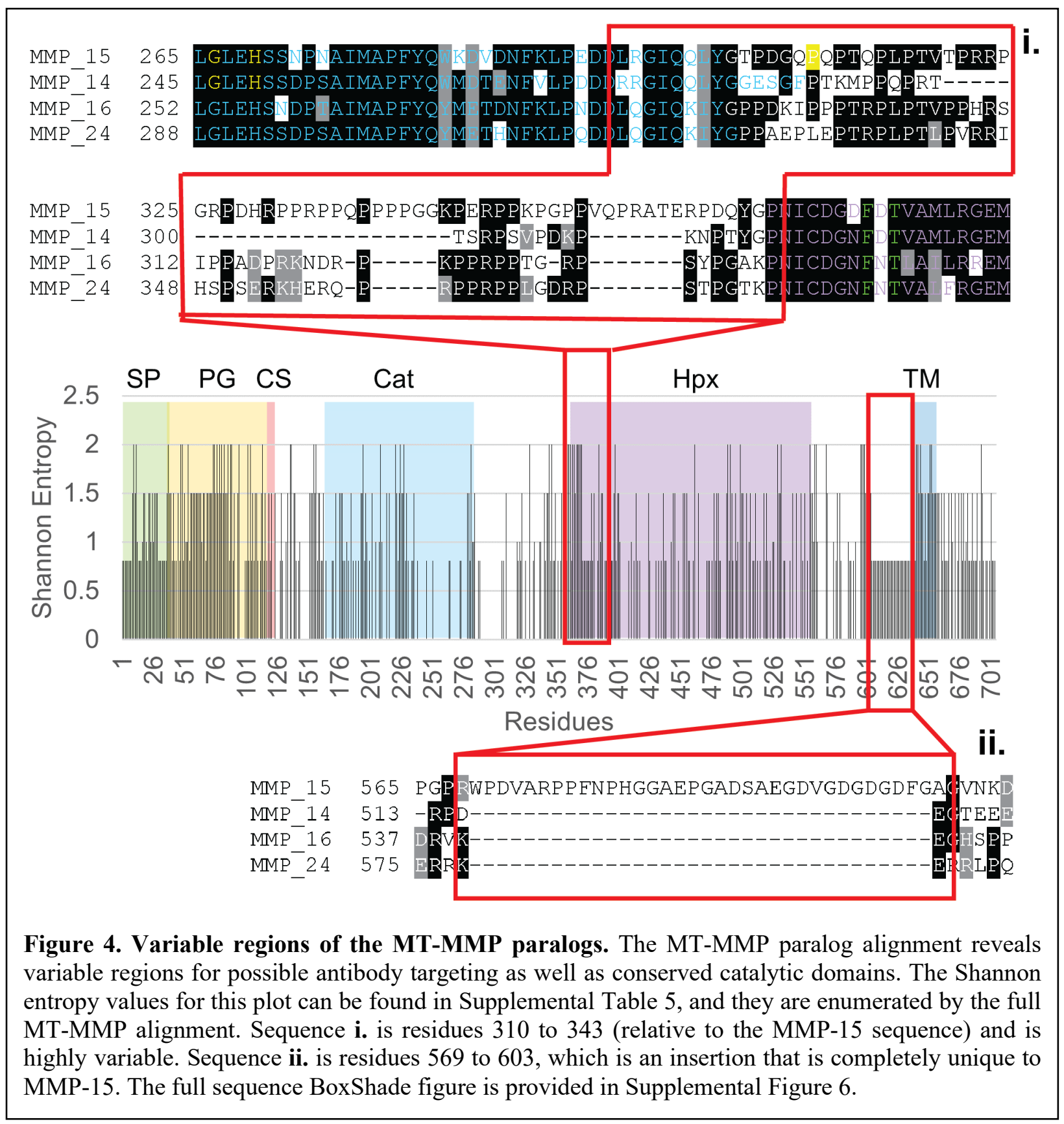


Specifically, two sequence regions, labeled as sequences i. and ii. in Figure 4, are shown in BoxShade alignments and have significant variability between paralogs. The two regions are amino acid residues 310 to 343 , and 569 to 603 . The first region, residues $310-343$, is in a proline-rich linker region between the catalytic and hemopexin domain extending into the $\mathrm{N}$-terminal portion of the hemopexin domain. Residues 569-603 are a stretch of 34 amino acids that are completely unique to MMP-15 compared to other MTMMP family members. This sequence appears to result from an insertion event in MMP-15 which did not occur in other MT-MMPs.

\section{Discussion}

In the development of cancer therapeutics, drug specificity has become integral to improving upon current treatments, as non-specific therapies may have significant toxicity. Immunotherapy utilizes the specificity of the immune system to target cancer cells, and the potential for identifying the most optimal target antigens and epitopes is increasingly important. In this work we investigate two comparisons of the potential antibody target MMP-15: i) MMP-15 orthologous sequences (across species) and ii) human MTMMP paralogous sequences, in order to identify conserved, and thereby likely important residues and to identify novel, unique epitopes of MMP-15, respectively. Sequence regions that are both essential to function and unique to the protein of interest have potential as epitopes for effective drug targeting, to impair target function and reduce toxicity, respectively. Conserved residues across MMP-15 orthologs, but divergent across MT-MMP paralogs, would not only optimize drug development by targeting residues conserved in function, but also may enable non-human efficacy and toxicity studies while minimizing offtarget effects by limiting binding to paralog epitopes. Here we identify such regions and suggest their viability as target epitopes.

In the mammalian MMP-15 ortholog analysis, the linker region before the transmembrane region is unannotated as a protein domain and is relatively variable. In addition, the high conservation of the MMP15 catalytic domain suggests that the MMP-15 substrate specificity between mammals is likely preserved. The docking of the substrate is dictated by the S1 pocket which varies in depth among MMPs and is the binding site for substrates (Cathcart, Nagase). In addition, our results support hemopexins as an important domain through their high conservation (Itoh, Butler). Previous work with MMP-14 demonstrated that the hemopexin domains are used for dimerization of MMP's, and hemopexins appear to be present in all MMPs (Nagase).

From the MT-MMP paralog analysis, we have identified key features across MT-MMP's as well as possible targeting regions with significant variability. There is an even distribution of variation across the entire sequence, however the placement of variation and the domains they are in may have significance. Not surprisingly, the catalytic domain has higher conservation, due to its importance in performing its function. The more variable regions of the catalytic domain may contain residues that control substrate specificity through altering the surface of the catalytic domain, such as the hydrophobic S1' pocket. Although these MT-MMP's are of the same family with their common transmembrane region, they may have diverse substrates (Nagase). The hemopexin domains were more diverse within the MT-MMP paralog comparison than the mammalian ortholog comparison, likely due to the fact that different types of MT-MMPs may have evolved divergent functions, and the hemopexin domain itself may have evolved unique dimerization components. 
The aim of this work is to identify possible epitope sequences. Variable regions between MT-MMPs is relevant to identifying more specific epitopes. In studies of MMP-14, complications of previous MMP small molecule inhibitors included unpredicted side effects due to the off-target inhibition of other MMPs (Overall, Giavazzi). If an antibody was directed to target highly conserved regions of paralogs, then it could bind to any MT-MMP and have off-target consequences. Therefore, regions that are unique to MMP-15 (versus other MMPs) are the potentially optimal epitopes. We identified residues 310-343 as highly variable, with high divergence from other sequences that might yield higher specificity of the antibody to MMP-15 only, lowering possible off-target toxicities. However, residues 569-603 appear to result from an insertional event and are completely distinctive to MMP-15, implying even higher potential specificity. This peptide appears to have a long extension which may allow MMP-15 a wider range of mobility on the cell surface than other MMPs. Alternatively, it may have an as yet undiscovered structure and function. Of considerable importance, these two regions may provide for optimal epitopes for antibody targeting. In this regard, high affinity, specific antibodies to these regions could be useful as therapeutics, diagnostics, or valuable research reagents.

Our results reveal that open-source bioinformatics tools could be used to identify conserved and diverse regions in the MMP-15 protein compared to other MMP-15 orthologs and other MT-MMP paralogs. These regions can be considered as potentially specific antigen epitopes of MMP-15 in consideration for antibody, drug, or diagnostic targeting. The next step is to use the sequences identified to discover and engineer high affinity monoclonal antibodies experimentally, which could be used in NSCLC or other diseases as therapeutics or diagnostics.

\section{Experimental Procedures}

We analyzed MMP-15 orthologous amino acid sequences from Homo sapiens (NP_002419.1), Mus musculus (NP_032635.1), Rattus norvegicus (NP_001099638.1), Bos taurus (NP_001178363.1), Canis lupus familiaris (XP_022270500.1), Pan troglodytes (XP_009429160.2), Equus caballus (XP_023492845.1). In a separate alignment, we analyzed four paralogous Homo Sapiens type I MT-MMPs: MMP-15, MMP-14 (NP_004986.1), MMP-16 (NP_005932.2), and MMP-24 (NP_006681.1), in the transmembrane (TM) paralog alignment. The amino acid sequences for both comparisons were procured from NCBI's protein data base. Both comparisons were aligned using Clustal Omega (Clustal), an online multiple sequence alignment software, which can be found at https:/www.ebi.ac.uk/Tools/msa/clustalo/. The program inputs were the FASTA sequences from NCBI (NCBI), and the output was specified as a Pearson/FASTA file. The results from Clustal Omega were also displayed with BoxShade using the BoxShade server at https://embnet.vital-it.ch/software/BOX form.html (BoxShade).

From the alignments, a variability plot was also performed using the Protein Variability Server at http://imed.med.ucm.es/PVS/, which calculates variability per residue (PVS). The variability plots were constructed for both the mammalian ortholog comparison and the MT-MMP paralog comparison through the Shannon entropy method, however for the orthologs the reference sequence was the consensus sequence while for the MT-MMP paralog comparison MMP-15 was the reference sequence. The Shannon entropy analysis is considered one of the most sensitive methods for estimating the diversity of a system (Shannon, Litwin). Additional plots were made for the variability of the catalytic domains and the hemopexin domains for both analyses with the same selections. 
After analyzing the sequences, we performed a structural analysis in the visualization program, Visual Molecular Dynamics (VMD) (Humphrey). Using the protein annotations from NCBI, domains were projected onto the 3-D structures according to residue. In addition, variable residues were investigated and visualized as described in each figure. The structures for both the mammalian species ortholog comparison and the TM-MMP paralog comparison are portions of various crystal structures of MMP-14, pdbs 3MA2 for the catalytic domain and $3 \mathrm{C} 7 \mathrm{X}$ for the hemopexin domains. Both crystal structures were from RCSB PDB (https://www.rcsb.org/), an online database for protein crystal structures (Berman, Grossman, Tochowicz). MMP-14 was used as a surrogate for MMP-15 (57.9\% identity) because there is currently no available crystal structure of MMP-15.

\section{Conflict of Interest}

The authors declare no conflicts of interest related to this manuscript.

\section{References}

1. Tyagi, A., Raina, K., Gangar, S., Kaur, M., Agarwal, R., and Agarwal, C. (2013) Differential effect of grape seed extract against human non-small-cell lung cancer cells: the role of reactive oxygen species and apoptosis induction. Nutr Cancer. 10.1080/01635581.2013.785003

2. Hallam, T. J., Wold, E., Wahl, A., and Smider, V. V. (2015) Antibody Conjugates with Unnatural Amino Acids. Mol. Pharmaceutics. 12, 1848-1862

3. Dotti, G., Gottschalk, S., Savoldo, B., and Brenner, M. K. (2014) Design and development of therapies using chimeric antigen receptor-expressing T cells. Immunol Rev. 257, 107-126

4. Ho, M.-Y., Tang, S.-J., Sun, K.-H., and Yang, W. (2011) Immunotherapy for Lung Cancers. $J$ Biomed Biotechnol. 10.1155/2011/250860

5. Steven, A., Fisher, S. A., and Robinson, B. W. (2016) Immunotherapy for lung cancer. Respirology. 21, 821-833

6. Pento, J. T. (2017) Monoclonal Antibodies for the Treatment of Cancer. Anticancer Res. 37, 59355939

7. Aldarouish, M., and Wang, C. (2016) Trends and advances in tumor immunology and lung cancer immunotherapy. J Exp Clin Cancer Res. 35, 157

8. Magdalena, B., and Andrzej, M. (2016) Differentiated Expression of Membrane Type Metalloproteinase (MMP-14, MMP-15) and Pro-MMP2 in Laryngeal Squamous Cell Carcinoma. A Novel Mechanism: Commentary on Research Study. J Med Surg Pathol. 10.4172/24724971.1000126

9. Tao, G., Levay, A. K., Gridley, T., and Lincoln, J. (2011) Mmp15 is a direct target of Snai1 during endothelial to mesenchymal transformation and endocardial cushion development. Dev Biol. 359, 209-221

10. Cathcart, J., Pulkoski-Gross, A., and Cao, J. (2015) Targeting Matrix Metalloproteinases in Cancer: Bringing New Life to Old Ideas. Genes Dis. 2, 26-34

11. Nagase, H., Visse, R., and Murphy, G. (2006) Structure and function of matrix metalloproteinases 
and TIMPs. Cardiovasc Res. 69, 562-573

12. Araki, Y., and Mimura, T. (2017) Matrix Metalloproteinase Gene Activation Resulting from Disordred Epigenetic Mechanisms in Rheumatoid Arthritis. Int J Mol Sci. 10.3390/ijms18050905

13. Itoh, Y. (2015) Membrane-type matrix metalloproteinases: Their functions and regulations. Matrix Biol. 44-46, 207-223

14. Fanjul-Fernández, M., Folgueras, A. R., Cabrera, S., and López-Otín, C. (2010) Matrix metalloproteinases: evolution, gene regulation and functional analysis in mouse models. Biochim Biophys Acta. 1803, 3-19

15. Löffek, S., Schilling, O., and Franzke, C.-W. (2011) Biological role of matrix metalloproteinases: a critical balance. European Respiratory Journal. 38, 191-208

16. Brew, K., Dinakarpandian, D., and Nagase, H. (2000) Tissue inhibitors of metalloproteinases: evolution, structure and function. Biochim Biophys Acta. 1477, 267-283

17. Overall, C. M., and López-Otín, C. (2002) Strategies for MMP inhibition in cancer: innovations for the post-trial era. Nat Rev Cancer. 2, 657-672

18. Zucker, S., Cao, J., and Chen, W.-T. (2000) Critical appraisal of the use of matrix metalloproteinase inhibitors in cancer treatment. Oncogene. 19, 6642-6650

19. Giavazzi, R., Garofalo, A., Ferri, C., Lucchini, V., Bone, E. A., Chiari, S., Brown, P. D., Nicoletti, M. I., and Taraboletti, G. (1998) Batimastat, a synthetic inhibitor of matrix metalloproteinases, potentiates the antitumor activity of cisplatin in ovarian carcinoma xenografts. Clin Cancer Res. 4, 985-992

20. Hotary, K. B., Yana, I., Sabeh, F., Li, X.-Y., Holmbeck, K., Birkedal-Hansen, H., Allen, E. D., Hiraoka, N., and Weiss, S. J. (2002) Matrix Metalloproteinases (MMPs) Regulate Fibrin-invasive Activity via MT1-MMP-dependent and -independent Processes. J Exp Med. 195, 295-308

21. Dornier, E., and Norman, J. C. (2018) Cancer cells with trapped nuclei cut their way through the extracellular matrix. Nat Commun. 9, 3954

22. Hotary, K., Allen, E., Punturieri, A., Yana, I., and Weiss, S. J. (2000) Regulation of cell invasion and morphogenesis in a three-dimensional type I collagen matrix by membrane-type matrix metalloproteinases 1, 2, and 3. J Cell Biol. 149, 1309-1323

23. Hotary, K., Li, X.-Y., Allen, E., Stevens, S. L., and Weiss, S. J. (2006) A cancer cell metalloprotease triad regulates the basement membrane transmigration program. Genes Dev. 20, 2673-2686

24. Ota, I., Li, X.-Y., Hu, Y., and Weiss, S. J. (2009) Induction of a MT1-MMP and MT2-MMPdependent basement membrane transmigration program in cancer cells by Snail1. Proc Natl Acad Sci U S A. 106, 20318-20323

25. Löffek, S., Zigrino, P., Steiger, J., Kurschat, P., Smola, H., and Mauch, C. (2006) Melanoma cellderived vascular endothelial growth factor induces endothelial tubulogenesis within fibrin gels by a metalloproteinase-mediated mechanism. Eur J Cell Biol. 85, 1167-1177

26. d'Ortho, M. P., Will, H., Atkinson, S., Butler, G., Messent, A., Gavrilovic, J., Smith, B., Timpl, R., Zardi, L., and Murphy, G. (1997) Membrane-type matrix metalloproteinases 1 and 2 exhibit broadspectrum proteolytic capacities comparable to many matrix metalloproteinases. Eur J Biochem. 250, $751-757$

27. Ito, E., Yana, I., Fujita, C., Irifune, A., Takeda, M., Madachi, A., Mori, S., Hamada, Y., Kawaguchi, N., and Matsuura, N. (2010) The role of MT2-MMP in cancer progression. Biochem Biophys Res 
Commun. 393, 222-227

28. Kobayashi, K., Nishioka, M., Kohno, T., Nakamoto, M., Maeshima, A., Aoyagi, K., Sasaki, H., Takenoshita, S., Sugimura, H., and Yokota, J. (2004) Identification of genes whose expression is upregulated in lung adenocarcinoma cells in comparison with type II alveolar cells and bronchiolar epithelial cells in vivo. Oncogene. 23, 3089-3096

29. Abraham, R., Schäfer, J., Rothe, M., Bange, J., Knyazev, P., and Ullrich, A. (2005) Identification of MMP-15 as an anti-apoptotic factor in cancer cells. J Biol Chem. 280, 34123-34132

30. Joshi, P., Jeon, Y.-J., Laganà, A., Middleton, J., Secchiero, P., Garofalo, M., and Croce, C. M. (2015) MicroRNA-148a reduces tumorigenesis and increases TRAIL-induced apoptosis in NSCLC. PNAS. 112, 8650-8655

31. Liu, Y., Xu, Y., Guo, S., and Chen, H. (2016) T cell factor-4 functions as a co-activator to promote NF-kB-dependent MMP-15 expression in lung carcinoma cells. Scientific Reports. 6, 24025

32. Clustal Omega $<$ Multiple Sequence Alignment $<$ EMBL-EBI [online] https://www.ebi.ac.uk/Tools/msa/clustalo/ (Accessed December 19, 2020)

33. Home - Protein - NCBI [online] https://www.ncbi.nlm.nih.gov/protein (Accessed December 19, 2020)

34. PVS: Protein Variability Server [online] http://imed.med.ucm.es/PVS/ (Accessed December 19, 2020)

35. Shannon, C. E. (1948) A Mathematical Theory of Communication. Bell System Technical Journal. 27, 379-423

36. Litwin, S., and Jores, R. (1992) Shannon Information as a Measure of Amino Acid Diversity. in Theoretical and Experimental Insights into Immunology (Perelson, A. S., and Weisbuch, G. eds), pp. 279-287, NATO ASI Series, Springer, Berlin, Heidelberg, 10.1007/978-3-642-76977-1_17

37. Humphrey, W., Dalke, A., and Schulten, K. (1996) VMD: visual molecular dynamics. J Mol Graph. 14, 33-38, 27-28

38. Berman, H. M., Westbrook, J., Feng, Z., Gilliland, G., Bhat, T. N., Weissig, H., Shindyalov, I. N., and Bourne, P. E. (2000) The Protein Data Bank. Nucleic Acids Res. 28, 235-242

39. Grossman, M., Tworowski, D., Dym, O., Lee, M.-H., Levy, Y., Murphy, G., and Sagi, I. (2010) The intrinsic protein flexibility of endogenous protease inhibitor TIMP-1 controls its binding interface and affects its function. Biochemistry. 49, 6184-6192

40. Tochowicz, A., Goettig, P., Evans, R., Visse, R., Shitomi, Y., Palmisano, R., Ito, N., Richter, K., Maskos, K., Franke, D., Svergun, D., Nagase, H., Bode, W., and Itoh, Y. (2011) The Dimer Interface of the Membrane Type 1 Matrix Metalloproteinase Hemopexin Domain. J Biol Chem. 286, 7587-7600

41. Butler, G. S., Will, H., Atkinson, S. J., and Murphy, G. (1997) Membrane-type-2 matrix metalloproteinase can initiate the processing of progelatinase A and is regulated by the tissue inhibitors of metalloproteinases. Eur J Biochem. 244, 653-657

42. Morpheus [online] https://software.broadinstitute.org/morpheus/ (Accessed December 19, 2020)

43. BoxShade Server [online] https://embnet.vital-it.ch/software/BOX_form.html (Accessed December 19, 2020) 